\title{
Development of a charge transfer space loop to improve adsorption performance in aerial electrostatic spray
}

\author{
Denan Zhao ${ }^{1,2}$, Yubin Lan ${ }^{1,2^{*}}$, Weiguo Shen ${ }^{3}$, Shizhou Wang ${ }^{1,2}$, Abhishek Dixit ${ }^{4}$ \\ (1. School of Agricultural Engineering and Food Science, Shandong University of Technology, Zibo 255000, Shandong, China; \\ 2. Shandong University of Technology Sub-center of National Center for International Collaboration Research on \\ Precision Agricultural Aviation Pesticides Spraying Technology, Zibo 255000, Shandong, China; \\ 3. Taicang Jingang Plant Protection Equipment Technology Co., Ltd., Taicang 215400, Jiangsu, China; \\ 4. School of Engineering, South China Agricultural University, Guangzhou 510642, China)
}

\begin{abstract}
In order to solve the problem of insufficient adsorption rate of droplets on the target back via aerial electrostatic spray, this study proposed a high-voltage electrostatic generator to charge the liquids in two isolated water tanks with positive and negative charges respectively. A charge transfer loop was developed in space between the aerial electrostatic spray system and the ground. This method greatly enhanced the adsorption performance under outdoor conditions that $16.7 \%$ droplets density increased on the target front, a nearly fourfold destiny increased on the target back compared with the conventional UAV spray system. The target back-to-front ratio of droplet density was improved from $6.1 \%$ to $25.7 \%$, which validated the satisfactory performance of the developed system.
\end{abstract}

Keywords: aerial electrostatic spray, electrostatic adsorption, charge transfer loop, back deposition, droplet, development DOI: $10.25165 /$ j.ijabe.20201305.5531

Citation: Zhao D N, Lan Y B, Shen W G, Wang S Z, Dixit A. Development of a charge transfer space loop to improve adsorption performance in aerial electrostatic spray. Int J Agric \& Biol Eng, 2020; 13(5): 50-55.

\section{Introduction}

The electrostatic spray has a smaller droplet size, which can make the droplets atomize evenly, increase target deposition, and improve contact opportunities with pests. This can greatly reduce the use of pesticides and improve the utilization rate ${ }^{[1-7]}$. Using fixed-wing aircraft or unmanned aerial vehicle (UAV) to spray pesticides is not restricted by terrain and has high operating efficiency, which is widely applied in many countries and regions in the world ${ }^{[8-11]}$. Combining these two technologies can bring benefits to agricultural production.

Many studies have been carried out in aerial electrostatic spray ${ }^{[12-15]}$. Kirk et al. ${ }^{[16,17]}$ conducted a lot of field tests on the aerial electrostatic spray system designed by Calton ${ }^{[18]}$, which demonstrated that aerial electrostatic spray can use about one-fifth pesticides of conventional spray to achieve the same insecticidal effect. Martin et al. ${ }^{[19]}$ proposed that when the charge-to-mass ratio was low, there was little difference in deposition between charged and non-charged spray. $\mathrm{Ru}$ et al. ${ }^{[20]}$ designed a UAV electrostatic spray system, and tests confirmed that the deposition density of droplets on the upper, middle and lower layers of rice all increased. Zhang et al. ${ }^{[21]}$ designed a six-rotor UAV electrostatic spray system, and used a metal ball attached to water-sensitive

Received date: 2019-11-20 Accepted date: 2020-06-15

Biographies: Denan Zhao, PhD candidate, research interest: agricultural mechanization engineering, Email: 714283646@qq.com; Weiguo Shen, General manager, research interest: electrostatic spray technology, Email: 124581362@qq.com; Shizhou Wang, PhD, Professor, research interest: precision agricultural aviation technology, Email: swang_1959@qq.com; Abhishek Dixit, Master candidate, research interest: agricultural engineering, Email: abhishekd95@stu.scau.edu.cn

*Corresponding author: Yubin Lan, $\mathrm{PhD}$, Professor, research interest: precision agriculture aviation technology. School of Agricultural Engineering and Food Science, Shandong University of Technology, Zibo 255000, Shandong, China. Tel: +86-533-2782718, Email: ylan@sdut.edu.cn. paper as a target to detect droplet deposition. However, the electrostatic adsorption force was not strong to overcome the gravity, so droplets were not deposited at the bottom of the metal ball. Zhou et al. ${ }^{[22]}$ designed an aerial electrostatic spray system based on the structure of the R44 helicopter. Performance test results showed that the uniformity of droplet deposition was improved and the droplet deposition on the back of the blade was achieved. However, the back deposition was caused by the turbulent airflow generated by the helicopter rotor, not by the electrostatic adsorption effect. In Zhou's aircraft spray test for controlling pine caterpillars, the aerial electrostatic spray system can reduce the use of pesticides by $5.22 \mathrm{~L} / \mathrm{hm}^{2}$, the mortality rate of pests has increased, and the pesticide efficacy has been further improved $^{[23]}$.

From the current research, it is obvious that the electrostatic force has improved the deposition density of droplets, and the fine droplets will also promote the adsorption and deposition of pesticides. But the disadvantage is that the droplets' adsorption performance of aerial electrostatic spray on the target back is insufficient, with room for improvement.

The current adsorption way between the charged droplets and the target is based on the principle of electrostatic induction ${ }^{[15]}$. A majority of researchers optimized the adsorption performance by increasing the charge-to-mass ratio of the droplets ${ }^{[24,25]}$. However, it turned out that a high charge-to-mass ratio did not mean strong adsorption and high droplets deposition on the target back. Studies have shown that the plants themselves carry bioelectricity ${ }^{[26]}$, which can promote the adsorption of charged droplets to the target back in theory. But the amount of bioelectric charge is always very small. Yu et al. ${ }^{[27]}$ suggested that plants can be artificially provided with electricity opposite to charged droplets, but this method was inefficient and limited on the ground.

To solve the problem of insufficient adsorption rate of droplets via aerial electrostatic spray, this study proposed to develop a 
charge transfer loop in space to improve adsorption performance.

\section{Materials and methods}

\subsection{Indoor test}

2.11 Aerial electrostatic spray system

A high-voltage electrostatic generator is shown in Figure 1. The input voltage is $12 \mathrm{~V}$. The working current is $300 \mathrm{~mA}$, which can generate and output $30 \mathrm{kV}$ positive and negative high-voltage simultaneously. The output current is about $1 \mathrm{~mA}$.

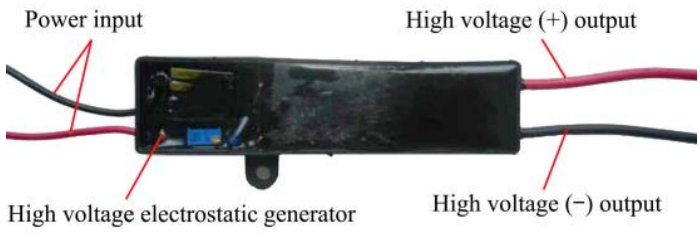

Figure 1 High-voltage electrostatic generator

There are three power supplies. Each of them has the power of $12 \mathrm{~V}$. Two water tanks of QWF80 plant-protection UAV were used and of propene polymer insulation material. The volume of each tank is $10 \mathrm{~L}$. Two diaphragm pumps were used to isolate the

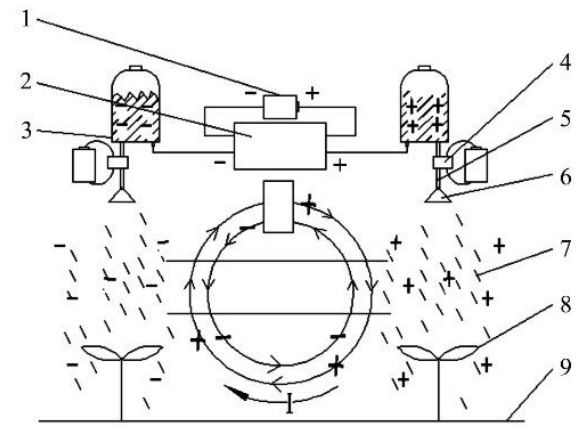

a. Schematic diagram of aerial electrostatic spray system charged liquid from the plunger and the pump cylinder to avoid the damage of high voltage, and thus protect the plunger and the pump cylinder. Two hollow conical nozzles are brass material and the flow of each nozzle is $0.225 \mathrm{~L} / \mathrm{min}$. A bracket was used to support and fix the test rig, and water pipes were used to connect the liquid in the water tank with the nozzle.

A start-stop device includes a remote control and electromagnetic relay device. The electromagnetic relay device includes three switches for the power supplies of the diaphragm pump (+), the diaphragm pump (-), and the high-voltage electrostatic generator, and it can be remotely controlled by the remote control. A suspension system, which includes two insulated nylon ropes and two fixed pulleys fixed to the roof. They were used to suspend the aerial electrostatic spray system, and to simulate the aircraft's ungrounded status.

The aforementioned components were connected and assembled according to the schematic diagram shown in Figure 2a. The physical diagram is shown in Figure $2 b$. The contact-charging method was used to charge the liquid in water tanks. The components connection diagram is shown in Figure 3.

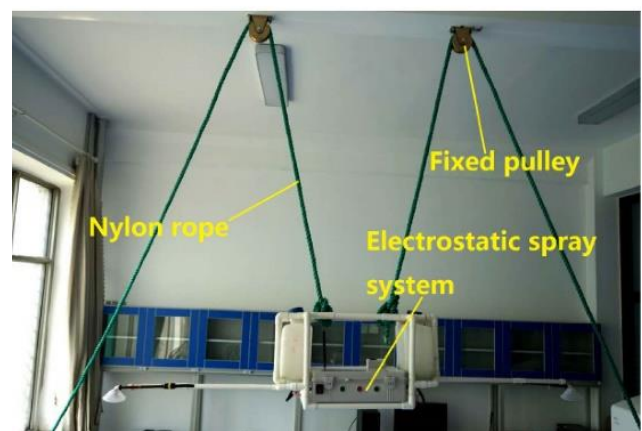

b. Aerial electrostatic spray system test bench

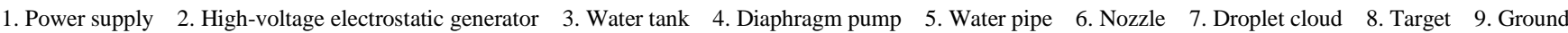

Figure 2 Aerial electrostatic spray system

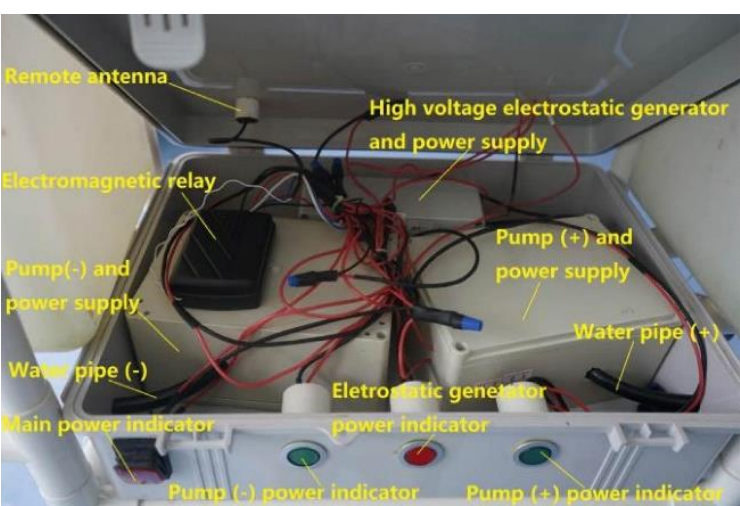

Figure 3 Components connection diagram

\subsubsection{Simulated target}

Simulated target method is a commonly used method for spray effect detection. As shown in Figure 4a and Figure 4b, each piece of paper was fixed by four wires, which were grounded through a hollow plastic tube to simulate the grounding of the field crop.

\subsubsection{Plant target}

Lanchong cinnamon and the blade in the horizontal state were selected as the target plant and the target blade. The pieces of coated paper with the same size were fixed at the same position on the front and back of the blade (Figure 4c). The specification of the coated paper was $80 \mathrm{~mm} \times 30 \mathrm{~mm}$.

\subsubsection{Droplets test method}

In the test, $5 \%$ of the food-grade tempting red additive aqueous solution was used as a tracer, and the spray effect was evaluated by the distribution of the dyed droplets on the front and back of the coated paper. Color images of the coated paper were scanned for qualitative analysis.

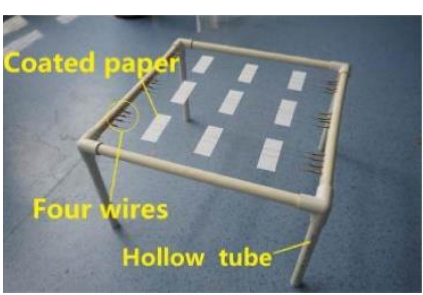

a. Simulated target

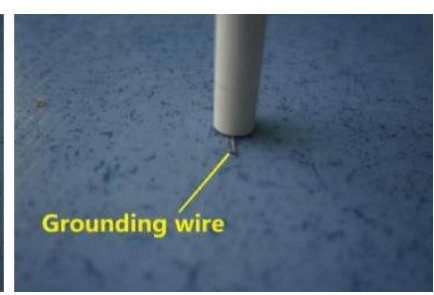

b. Grounding wire

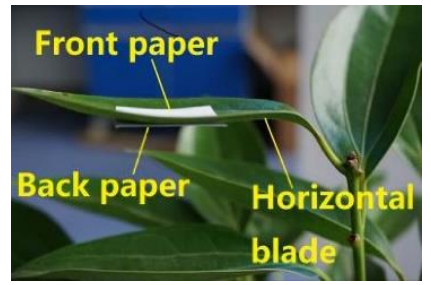

c. Plant target

Figure 4 Targets of indoor test

\subsubsection{Test conditions}

Test condition A: the indoor temperature was $17^{\circ} \mathrm{C}-19^{\circ} \mathrm{C}$ with dry ground. The air humidity was $39 \%-42 \%$. Based on the measurement, the laboratory ground was non-conductive.

Test condition B: the indoor temperature was $17^{\circ} \mathrm{C}-19^{\circ} \mathrm{C}$. The ground of the laboratory was sprinkled with water. The air humidity was $55 \%-61 \%$, and the ground under this condition was 
determined to be conductive based on measurement.

\subsubsection{Indoor test design}

Six treatments of aerial electrostatic spray indoor tests were performed. The spray time of each treatment was $2.0 \mathrm{~s}$ based on the dosage of aerial conventional spray. The contact-charging voltage was $30 \mathrm{kV}$, and the height of the nozzles from targets was $0.5 \mathrm{~m}$. The schematic diagram is shown in Table 1. The specific treatment method is as follows.

Treatment 1: (+) spray, simulated target, test condition A;

Treatment 2: (-) spray, simulated target, test condition A;

Treatment 3: ( \pm ) sprays simultaneously, simulated target, test condition A;

Treatment 4: ( \pm ) sprays simultaneously, simulated target, test condition A. The simulated targets in two sides were connected by wires and positively-connected in series with high-voltage micro-ampere meter. Note: when current flows move through the ammeter, the ammeter is positively-connected with a positive value of " $+X$ ". When the ammeter is negatively-connected, it displays a negative value of " $X$ ". " $X$ " represents the magnitude of the current value, and " \pm " represents the direction of the current. A positive connection means that the upper terminal of the ammeter is connected to the grounding wire of the simulated target under the (+) spray, and the lower terminal is connected to the grounding wire of the simulated target under the (-) spray. A negative connection has the opposite meaning.

Treatment 5: ( \pm ) sprays simultaneously, simulated target, test condition $\mathrm{B}$;

Treatment 6: $( \pm)$ sprays simultaneously, plant target, test condition $\mathrm{B}$.

\section{Table 1 Design of six treatments of aerial electrostatic spray of indoor test}

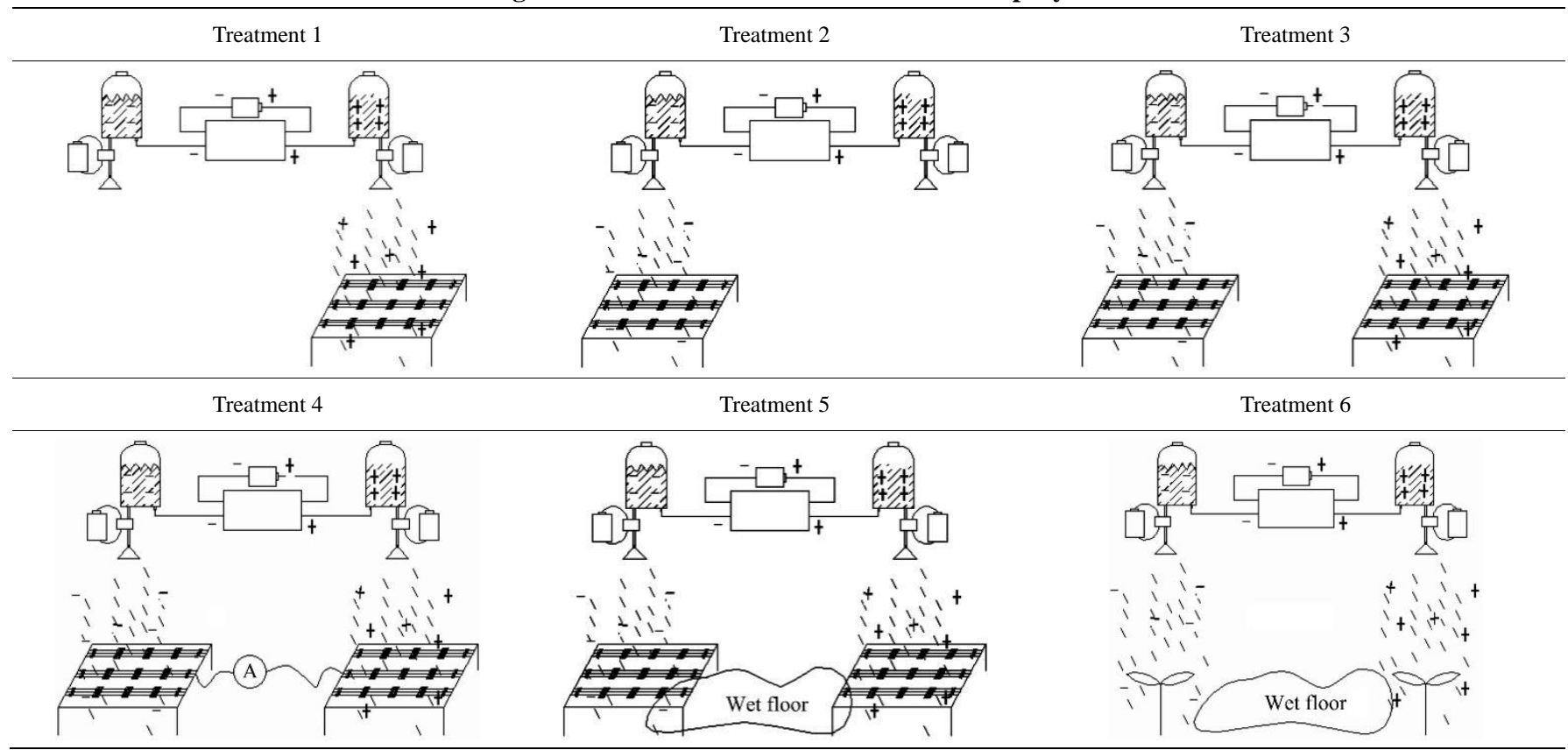

\subsection{Outdoor UAV test}

2.2.1 Aerial electrostatic spray system loaded on the UAV

As shown in Figure 5, the designed aerial electrostatic spray system was loaded on the high-tech M45 plant protection UAV, and the matching design was carried out according to its structural characteristics. The specific parameters of the UAV and electrostatic spray system are shown in Table 2. In order to increase the spray flow, each nozzle has a double nozzle design.

\subsubsection{Simulated target}

The design of the simulated target should ensure that the target can be connected in series in the entire charge transfer loop, so the simulated target was required to be grounded. PVC pipes and multi-directional clips were insulators, and they had a large difference in conductivity from plants and cannot be grounded, so they were not desirable. Based on the above considerations, the simulated target of the outdoor test (Figure 6) was designed as follows: the basic structure was a PVC pipe and a multi-directional clip, and the PVC pipe was inserted into the ground about $10 \mathrm{~cm}$. Punch a hole with a diameter of $8 \mathrm{~mm}$ at one end of an iron piece with $80 \mathrm{~mm}$ long and $40 \mathrm{~mm}$ wide. Put two pieces of water-sensitive paper on the upper and lower sides of the iron piece, and then be fixed to the clip. Through the hollow PVC pipe, the wire connected the hole in the iron piece to the ground. Finally, a long metal pin was connected to the wire and inserted into the soil to improve grounding stability. The iron piece was in a horizontal state, and its height from the ground was $1.0 \mathrm{~m}$.

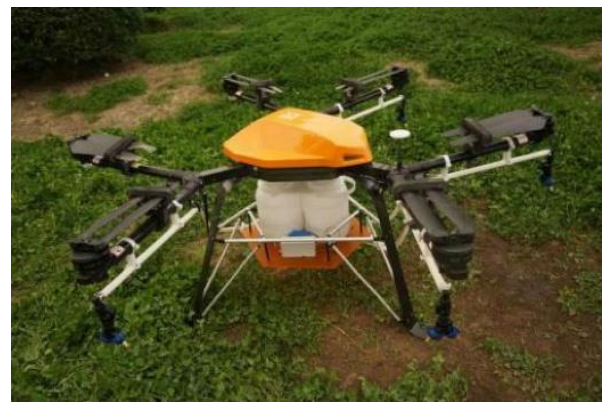

Figure 5 Aerial electrostatic spray system loaded on the UAV

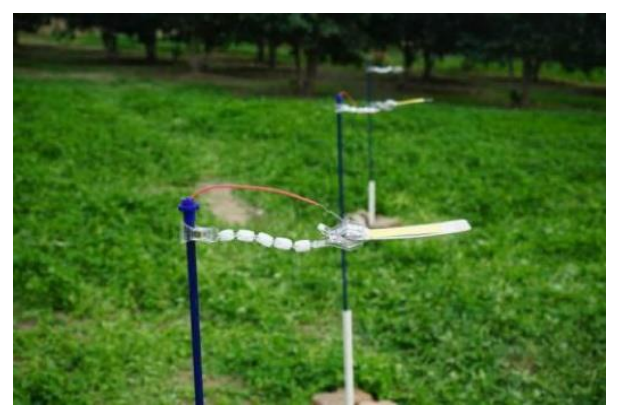

Figure 6 Simulated target design of outdoor test 
Table 2 Basic parameters of aerial electrostatic spray system loaded on the UAV

\begin{tabular}{|c|c|}
\hline Parameters & Values \\
\hline Maximum takeoff weight $/ \mathrm{kg}$ & 47 \\
\hline Load weight $/ \mathrm{kg}$ & 20 \\
\hline Battery capacity/mA $\cdot \mathrm{h}$ & 24000 \\
\hline Voltage of electrostatic generator/kV & 30 \\
\hline Dimensions (diameter $\times$ height, folded state) $/ \mathrm{mm}$ & $1150 \times 767$ \\
\hline Dimensions (diameter $\times$ height, expanded state) $/ \mathrm{mm}$ & $1800 \times 767$ \\
\hline Flight speed $/ \mathrm{m} \cdot \mathrm{s}^{-1}$ & $0-6$ \\
\hline Flight height $/ \mathrm{m}$ & $2-5$ \\
\hline Spray width/m & $6-8$ \\
\hline Number of nozzles & 8 \\
\hline Number of rotors & 6 \\
\hline Total flow/L $\cdot \min ^{-1}$ & $1.8-2.6$ \\
\hline
\end{tabular}

\subsubsection{Outdoor test design}

Three grounded targets were arranged on one straight line, and the distance between each target was $1.2 \mathrm{~m}$. The UAV was flying in the direction perpendicular to the straight line where the targets were located, with a flight speed of $4 \mathrm{~m} / \mathrm{s}$ and a flight height of $3.5 \mathrm{~m}$ (according to the general flight parameters of Gaoke M45 plant protection UAV). Two independent experiments were conducted. The first group was aerial conventional spray mode, and the second group was aerial electrostatic spray mode. Each group of tests has been repeated three times. Define the target back-to-front ratio of droplet density (back-to-front ratio, BFR in short) as an index for evaluating the droplet adsorption performance.

\section{Theory}

As shown in Figure 7, a high-voltage electrostatic generator is used to generate and output the positive and negative charges simultaneously. The positive and negative charges flow through the isolated water tanks and water pipes respectively. Then they are ejected from nozzles on both sides. After that, the two wet droplet clouds formed by the continuously falling droplets are connected to the ground. Both positive and negative charges flow into the ground and attract each other, making reverse movements in opposite directions, thereby forming a loop of charge transfer in space. This reverse movement at the interface between the target and the droplet cloud appears in the status of the mutual attraction between the charged droplets and the target. In essence, the reverse movement of positive and negative charges generates a current. That is, the positive output of the high-voltage electrostatic generator positively charged the target under the (-) spray through the connection of the liquid pipe, the wet droplet cloud and the ground. The negative output also negatively charged the target under the (+) spray through the symmetrical route. In this way, targets are finally charged by the grounding of droplet clouds. Theoretically, this way of actively charging plants gives plants and droplet clouds a stronger adsorption force compared with plants' induction charging of traditional aerial electrostatic spray.

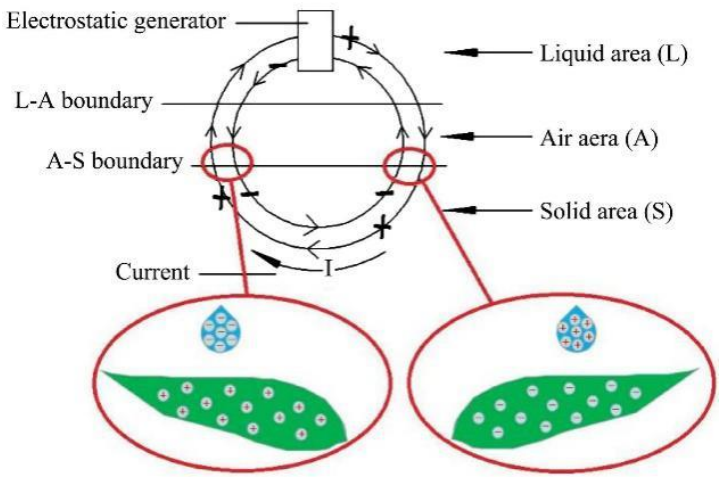

Figure 7 Electrostatics physical model of aerial electrostatic spray based on charge transfer space loop

\section{Results and discussion}

\subsection{Indoor test results and discussion}

The indoor test results of six treatments were shown in Table 3 . Among them, treatments 1-5 were the scanned images of the front and back of the same coated paper, and treatment 6 was the scanned images of two coated papers on the front and back of the horizontal plant blade. In addition, the display value of the ammeter was $+(1-2) \mu \mathrm{A}$ (unstable) in treatment 4

Table 3 Inoor test results of six treatments

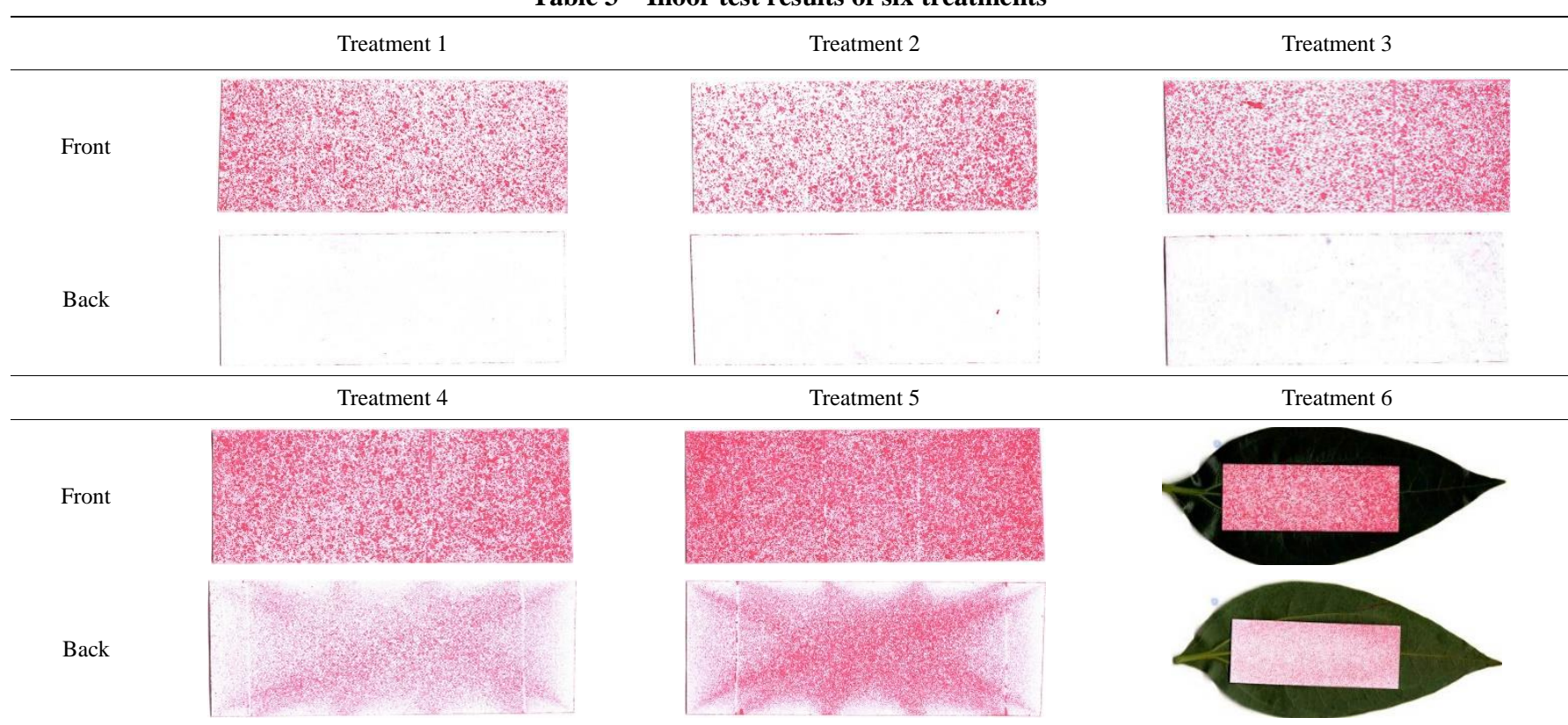

By comparing treatment 1 with treatment 2, there was no significant difference in the deposition of droplets on the back of coated paper regardless it was a positive or negative electrostatic spray. The deposition density was close to 0 in both treatments, 
indicating that the inductive adsorption force between the charged droplets and the target was insufficient to overcome the gravity barrier and achieve good back deposition under such conditions.

The motion of the droplets was analyzed to distinguish the difference between the aerial electrostatic spray and the ground electrostatic spray (Figure 8). As shown in Figure 8a, the droplets in the aerial electrostatic spray moved downward, which required an angle change of movement direction by $90^{\circ}-180^{\circ}$ to enable droplets to move to the back of the target. However, when the ground electrostatic spray was sprayed next to the plant, as shown in Figure 8b, it was likely to be sprayed parallel to the blade or sprayed upward. In order to reach the back of the target, the movement direction needs to be changed from $0^{\circ}$ to $90^{\circ}$. Therefore, the electrostatic adsorption force of aerial electrostatic spray and ground electrostatic spray for target back deposition varies greatly. For aerial electrostatic spray in horizontal target, the adsorption force generated by electrostatic induction is insufficient, which may be the reason why there is very little deposition on the back of the treatment 1 and treatment 2 . a. Vertical downward spray

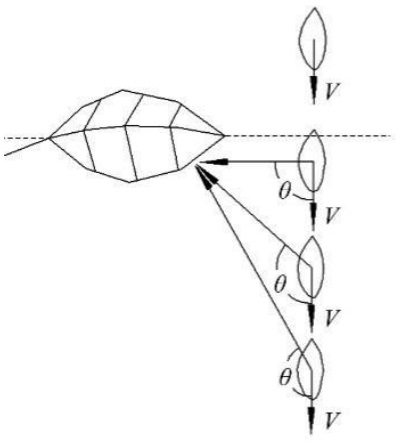

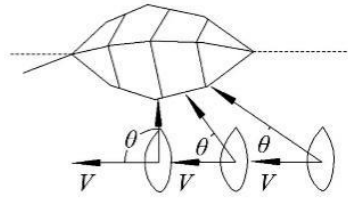

b. Horizontal spray
Figure 8 Motion analysis of vertical downward spray and horizontal spray

Compared to treatments 1 and 2, treatment 3 did not show obvious electrostatic adsorption advantages on the back of the target. This might be because the ground was not electrically conductive, and '+' and '-' charges cannot continue to move to the target below the electrostatic spray with the opposite polarity.

Compared to treatment 3 , the target back deposition of treatment 4 showed a qualitative change and was significantly improved. The difference between treatments 3 and 4 was if there was a condition for the encounter of positive and negative charges. The ground in treatment 3 was not conductive so that the droplets on the two sides cannot continue moving to the opposite side after falling on the ground but to gather separately. However, in treatment 4 , the targets on two sides were connected by a wire. Two charges can move to the opposite target through the wire after being grounded by the droplet clouds, and then charge the plants to attract the droplets with the opposite polarity for the downward movement. Moreover, in treatment 4, the display value of the high-voltage micro-ampere meter was $+(1-2) \mu \mathrm{A}$, which proved that there was indeed current. And very importantly, the current value was "+" instead of "-", indicating that the direction of current was from the part below the $(+)$ electrostatic spray to the part below the (-) electrostatic spray rather than the opposite, which showed there was a reverse movement of positive and negative charges. Therefore, it demonstrated that the use of droplet clouds grounding was a method of connection and the formation of the charge transfer loop was the key for adsorption, which enable plants to carry electricity of opposite polarity to the droplets.

The back deposition advantage of treatments 4-5 was much significant than that of treatments 1-3. Based on the analysis, the reason was that treatments 4 and 5 created the conditions for charge transfer. However, there were still some gaps in the amount of deposition between treatments 4 and 5 . The possible reason was that the conductivity between the wire and the wet ground was different.

It was worth mentioning that the target back of treatments 4 and 5 showed a similar characteristic of 'spike-like deposition effect'. The edge of the test paper produced a lot of unevenly distributed "spike" protrusions (as shown in Figure 9). This may be related to the arrangement of the wires of the simulated target, which affects the electric field arrangement and causes this peculiar phenomenon.

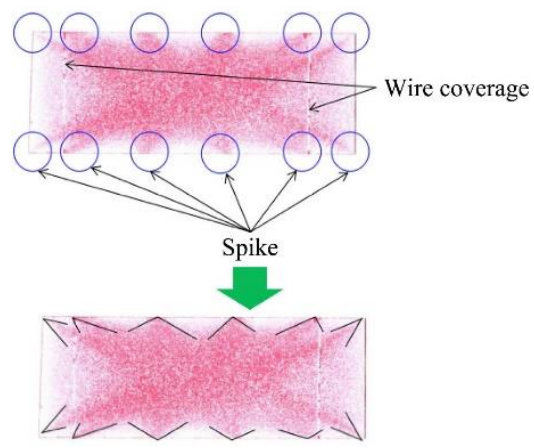

Figure 9 Spike-like deposition effect

There was a possibility that the wire could attract the droplets more easily. This assumption can be explained by the back deposition of the treatments 1-3. Although the simulated targets of treatments 1-3 were the same as that of treatments 4-5, the former did not cause a large amount of droplet deposition due to the presence of the wire. The presence of the wires only affected the uniformity of the distribution, but it was not the root cause of a large amount of droplet deposition on the back of the target.

The difference between treatments 5 and 6 was caused by the difference in the target. The back deposition image of treatment 6 was relatively more uniform compared with treatment 5 . The possible reason was that the electric field distribution around the leaf in treatment 6 was relatively uniform and was not disturbed by the wires.

By comparing the back deposition images of treatments 4-6 with that of treatments $1-3$, and by the formation of current as well as its direction in treatment 4 , it was proved that the method of building a charge transfer space loop to charge the targets can greatly increase the target back deposition with a significant adsorption effect. This differs from the electrostatic induction of the target in the strength of the adsorption force.

\subsection{Outdoor test results and discussion}

From the test results, it can be concluded that the droplet deposition density on the front and back sides of the target were both improved when the electrostatics were turned on. The droplet density on the front of the target increased from 56.8 droplets $/ \mathrm{cm}^{2}$ to 66.1 droplets $/ \mathrm{cm}^{2}$ on average, resulting in a $16.4 \%$ increase (as shown in Figure 10). And on the back, it has increased from 3.5 droplets $/ \mathrm{cm}^{2}$ to 17 droplets $/ \mathrm{cm}^{2}$ on average, resulting in a nearly fourfold increase, which was much significant than that on the front. BFR increased from $6.1 \%$ to $25.7 \%$ on average, and the adsorption performance of droplets was significantly improved.

But compared with the indoor adsorption phenomenon, the outdoor adsorption performance has been weakened. There are two possible reasons. Firstly, the rotor wind of the UAV will 
create resistance to the droplets which might be adsorbed to the back of the target. Secondly, limited by the structure of the Gaoke M45 UAV, the distance between the nozzles is too close, which will lead to the neutralization of the droplet clouds with positive and negative charges, making the droplets' charge-to-mass ratio lower and affecting the formation of charge transfer space loop.

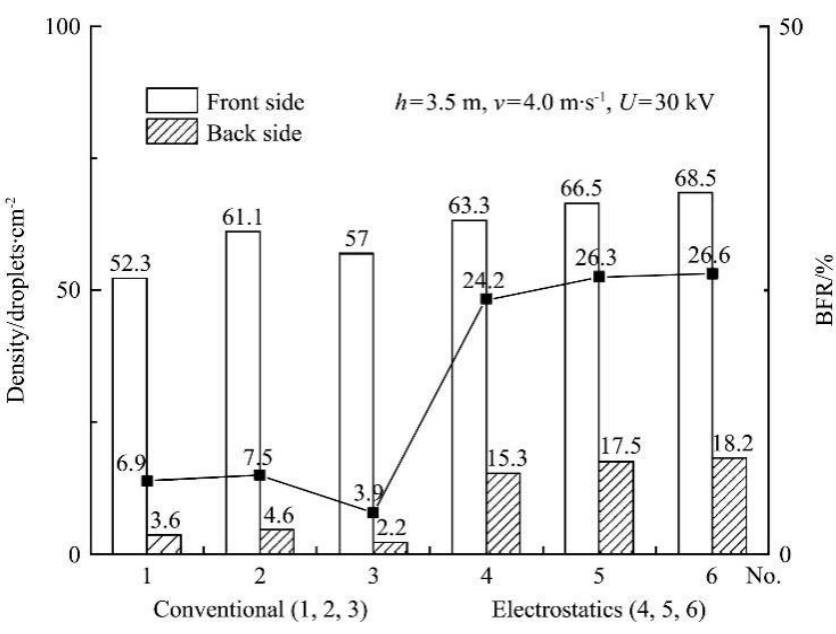

Figure 10 Outdoor test results

\section{Conclusions}

In this study, an aerial electrostatic spray test bench and a UAV electrostatic spray system were designed, and the method of developing a charge transfer loop in space to improve the droplet adsorption performance was proved to be feasible. Under indoor conditions without rotor wind, this adsorption performance was particularly obvious, and it produced a strong spike-like distribution effect. Under outdoor conditions, this method was also applicable, which can increase the droplet density on the target back by nearly fourfold compared with conventional spray. However, the adsorption performance will also be affected by the rotor wind, UAV's structure and other factors.

\section{Acknowledgements}

This study was financially supported by the Shandong Province Introduced the Top Talents 'One Case One Discussion' Special Funded Project (2018.01-2021.12); the Central Government Guided Local Science and Technology Development Special Fund 'Precision Agricultural Aviation Technology and Equipment Research and Development' Funded Project (2017.1-2019.12); the Shandong Province Dry Farming Intelligent Agricultural Machinery Equipment Collaborative Innovation Center Funded Project (2017.7-2020.12), and the Zibo Science and Technology Development Plan Funding Project (Grant No. 2018kj010073).

\section{[References]}

[1] Law S E. Electrostatic pesticide spraying: concepts and practice. IEEE Transactions on Industry Applications, 1983; IA-19(2): 160-168.

[2] Patel K M. Technological improvements in electrostatic spraying and its impact to agriculture during the last decade and future research perspectives-A review. Engineering in Agriculture, Environment and Food, 2016; 9(1): 92-100.

[3] Law S E. Agricultural electrostatic spray application: a review of significant research and development during the 20th century. Journal of Electrostatics, 2001; 51-52: 25-42.

[4] Lan Y B, Zhang H Y, Wen S, Li S H. Analysis and experiment on atomization characteristics and spray deposition of electrostatic nozzle. Transactions of the CSAM, 2018; 49(4): 130-139. (in Chinese)

[5] Singh M, Ghanshyam C, Mishra P K, Chak R. Current status of electrostatic spraying technology for efficient crop protection. AMA-Agricultural Mechanization in Asia Africa and Latin America, 2013; 44(2): 46-53.

[6] Wang Z T, Zhang Y H, Guo T Y, Zhu Z H, Wang X Y, Wen J L. Experimental study on size and velocity of charged droplets. Procedia Engineering, 2015; 126: 522-526.

[7] Zhu H P, Xian F S, Gao L R. Summary of research on electrostatic spraying technique theory and its applications. Transactions of the CSAM, 1989; 2: 53-59. (in Chinese)

[8] Lan Y B, Thomson S J, Huang Y B, Hoffmann W C, Zhang H H. Current status and future directions of precision aerial application for site-specific crop management in the USA. Computers \& Electronics in Agriculture, 2010; 74(1): 34-38.

[9] Xue X Y, Lan Y B. Agricultural aviation applications in USA. Transactions of the Chinese Society of Agricultural Machinery, 2013; 44(5): 194-201. (in Chinese)

[10] Lan Y B, Chen S D, Fritz B K. Current status and future trends of precision agricultural aviation technologies. Int J Agric \& Biol Eng, 2017 10(3): $1-17$.

[11] Thomson S J, Smith L A, Hanks J E. Evaluation of application accuracy and performance of a hydraulically operated variable-rate aerial application system. Transactions of the ASABE, 2009; 52(3): 715-722.

[12] Inculet I I, Fischer J K. Electrostatic aerial spraying. IEEE Transactions on Industry Applications, 1989; 25(3): 558-562.

[13] Zhang Y L, Lan Y B, Bradley K F, Xue X Y. Development of aerial electrostatic spraying systems in the United States and applications in China. Transactions of the CSAE, 2016; 32(10): 1-7. (in Chinese)

[14] Ru Y. Research on aerial pesticide electrostatic spraying system and its application. PhD dissertation. Nanjing Forestry University, 2009; 132p. (in Chinese)

[15] Carlton J B, Bouse L F. Spray deposit sampling technique to evaluate electrostatic aerial spray-charging. Transactions of the ASAE, 1978; 21(1): 0002-0005.

[16] Kirk I W, Harp S J, Wiese A M. Aerial sprays of fipronil for control of boll weevil. In: 2000 Proceedings Cotton Conferences Volume 2, San Antonio, 2000; pp.1281-1283.

[17] Latheef M A, Carlton J B, Kirk I W, Hoffmann W C. Aerial electrostatic-charged sprays for deposition and efficacy against sweet potato whitefly (Bemisia tabaci) on cotton. Pest Management Science, 2009; 65(7): 744-752.

[18] Carlton J B. Technique to reduce chemical usage and concomitant drift from aerial sprays. Patent of the United States, 5975425.1999-11-02.

[19] Martin D E, López J D, Hoffmann W C, Fritz B K, Lan Y B. Field evaluation of spinosad aerial applications for thrips control on cotton. Southwestern Entomologist, 2007; 32(4): 221-228.

[20] Ru Y, Jin L, Jia Z C, Bao R, Qian X D. Design and experiment on electrostatic spraying system for unmanned aerial vehicle. Transactions of the CSAE, 2015; 31(8): 50-55. (in Chinese)

[21] Zhang Y L, Lian Q, Zhang W. Design and test of a six-rotor unmanned aerial vehicle (UAV) electrostatic spraying system for crop protection. Int J Agric \& Biol Eng, 2017; 10(6): 68-76.

[22] Zhou H P, Ru Y, Shu C R, Zheng J Q, Zhu H P. Design and experimental research of aerial electrostatic spray system applied to helicopter. In: The 2nd China Forestry Academic Conference, 2009; pp.30-37. doi: 10.13031/2013.27246. (in Chinese)

[23] Zhou H P, Ru Y, Shu C R, Jia Z C. Improvement and experiment of aerial electrostatic spray device. Transactions of the CSAE, 2012; 28(12): 7-12. (in Chinese)

[24] Law S E. Embeded-electrode electrostatic-induction spray charging nozzle: theoretical and engineering design. Transaction of ASAE, 1978; 21(6): 1096-1104.

[25] Patel M K, Praveen B, Sahoo H K, Patel B, Kumar A, Singh M, et al. An advance air-induced air-assisted electrostatic nozzle with enhanced performance. Computers \& Electronics in Agriculture, 2017; 127: 280-288.

[26] Davies E. Action potentials as multifunctional signals in plants: a unifying hypothesis to explain apparently disparate wound responses. Plant Cell \& Environment, 1987; 10(8): 623-631.

[27] Yu D Y, Xian F S, Gao L R. Preliminary research on effect of charge plant on ultra-low-volume electrostatic spraying. Journal of Jiangsu University (National Science Edition), 1985; 1: 14-24. (in Chinese) 